\title{
Carrier Protein-Free Chemo-Enzymatic Synthesis of Arylomycin A2 and Structural Characterization of the Cytochrome P450 AryC
}

\author{
Hülya Aldemir, ${ }^{\dagger, \ddagger}, \|$ Shuangjie Shu, ${ }^{\dagger, \ddagger}, \|$ Francoise Schaefers, ${ }^{\ddagger}$ Hanna Hong, ${ }^{\ddagger}$ René Richarz, ${ }^{\dagger}$ Sabrina \\ Harteis, ${ }^{\ddagger}$ Manuel Einsiedler, ${ }^{\dagger}$ Tobias M. Milzarek, ${ }^{\dagger}$ Sabine Schneider, ${ }^{\perp, *}$ Tobias A. M. Gulder ${ }^{\dagger, *}, *$ \\ ${ }^{\dagger}$ Chair of Technical Biochemistry, Technical University of Dresden, Bergstraße 66, 01069 Dresden, Germany. \\ * Biosystems Chemistry, Faculty of Chemistry, Technical University of Munich, Lichtenbergstraße 4, 85748 Garching, Ger- \\ many. \\ ${ }^{\perp}$ Department of Chemistry, Ludwig-Maximillians-University Munich, Butenandtstraße 5-13, 81377 Munich, Germany. \\ E-Mail: sabine.schneider@cup.lmu.de; tobias.gulder@tu-dresden.de.
}

\begin{abstract}
Most antibiotics in clinical use are inspired by natural products. Prominent examples are the glycopeptides, such as vancomycin. These compounds contain biaryl- and biaryl-ether bonds that are crucial for biological activity and biosynthetically get installed by dedicated cytochrome P450 enzymes. The application of these biocatalysts in the chemo-enzymatic synthesis of novel glycopeptides has been impeded by their strict requirement for peptidyl carrier-protein (PCP) bound substrates and additional $\mathrm{X}$ domain interactions. This necessitates equimolar amounts of protein tethers, precluding truly catalytic applications. We describe the functional and structural evaluation of AryC, the cytochrome P450 performing biaryl coupling in biosynthetic arylomycin assembly, and its application in the chemo-enzymatic synthesis of arylomycin A2. AryC efficiently converts free substrates without the requirement of any protein interaction partner, likely enabled by a strongly hydrophobic cavity at the surface of AryC pointing to the substrate tunnel. The resulting reactivity of AryC is unprecedented in cytochrome P450-mediated biaryl construction in non-ribosomal peptides, in which PCP-tethering so far was crucial both in vivo and in vitro. Our work thus provides a basis for the development of general biocatalytic platforms for the efficient biocatalytic synthesis of biaryl peptide antibiotics.
\end{abstract}

Ever since their discovery, glycopeptide antibiotics have fascinated researchers across disciplines. Their challenging structures have inspired many innovative synthetic routes, ${ }^{1}$ an active area of research until to date. ${ }^{2}$ Likewise fascinating is their biosynthesis: the individual amino acid building blocks - including the prototypical hydroxyphenyl glycine (HPG) units whose assembly is also encoded in the respective biosynthetic gene clusters ${ }^{3}$ - are linked by nonribosomal peptide synthetases (NRPS). ${ }^{4}$ The resulting linear peptide precursor is oxidatively cross-linked while still bound to the terminal peptidyl carrier protein (PCP) of the NRPS, catalyzed by dedicated cytochrome P450 enzymes. This establishes the rigid polycyclic peptide core structure, e.g. in vancomycin (aglycon structure 1, Figure 1). ${ }^{5}$ The mechanism and timing of this process was illuminated by in-depth in vivo (predominantly by Suessmuth, Stegmann, and Wohlleben $)^{6}$ and in vitro (Robinson) ${ }^{7}$ studies. In recent landmark work, Cryle and coworkers furthermore established that a so-called X-domain, a previously uncharacterized domain found directly upstream of the final thioesterase in glycopeptide NRPSs, is required to orchestrate enzymatic crosslinking by the cytochromes. ${ }^{8}$ This finding also set the stage for the first successful in vitro reconstitution of the entire oxidative cross-linking cascade to chemo-enzymatically prepare glycopeptide aglycons and modified analogs. ${ }^{9}$ This large body of impressive work conducted over decades of research ultimately established the biosynthetic logic of glycopeptide assembly, ${ }^{10}$ but also revealed many challenges in applying the oxidative biosynthetic machinery in vitro as biocatalytic system. Most importantly, the necessity of equimolar amounts of coenzyme A (for precursor activation allowing PCP tethering) and equimolar amounts of $\mathrm{PCP}-\mathrm{X}$ didomain protein as crucial template for substrate recognition by the cross-linking $\mathrm{P} 450$ s precludes a truly (bio)catalytic approach.

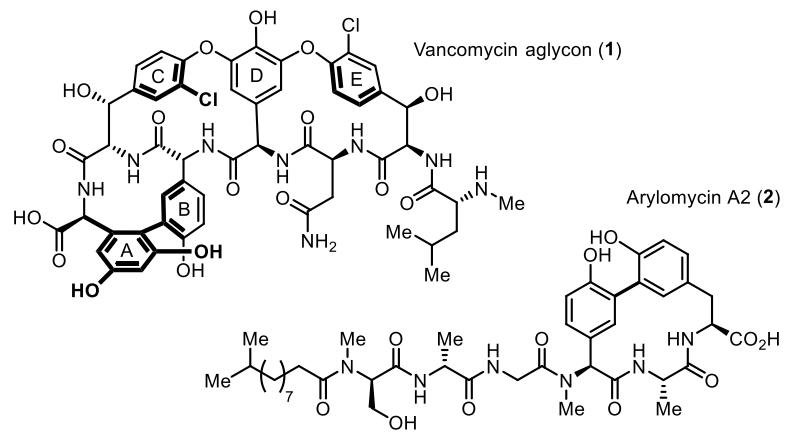

Figure 1. Structures of vancomycin aglycon (1) and arylomycin A2 (2).

A likewise exciting class of biaryl-containing peptides (BCPs) are the arylomycins, such as arylomycin A2 (2). ${ }^{11}$ The arylomycins bind to and thereby inhibit bacterial type I signal peptidase (SPase), an essential enzyme of the secretory pathway that is not addressed by any approved drug and thus represents a promising new antibiotic target. ${ }^{12}$ Owing to these interesting properties, the arylomycins have attracted strong interest from the synthetic community. The first total syntheses relied on the preparation of linear peptide precursors followed by macrocylization by Suzuki-Miyaura reaction. ${ }^{13}$ While these approaches also enabled first efforts towards optimization of arylomycin antibiotic potential, ${ }^{14}$ even leading to congeners with efficient Gram-negative activity, ${ }^{15}$ particularly the macrocyclization step left room for synthetic improvement. Further work provided elevated yields to the macrocyclic arylomycin core by macrolactamization ${ }^{16}$ and improved Suzuki-Miyaura macrocyclization. ${ }^{17}$ However, all these approaches still require the functionalization of the aromatic amino acid precursors to facilitate Pd-me- 
diated cross-coupling chemistry. Romesberg and Baran enabled access to the macrocycle by $\mathrm{C}-\mathrm{H}$ functionalization of the unmodified aromatic amino acids by copper-mediated oxidative phenol coupling. ${ }^{18}$ This elegant approach, however, requires two equivalents of the copper reagent. Most recent work achieved catalytic oxidative cross-coupling using $\mathrm{Fe}[\mathrm{TPP}] \mathrm{Cl}$, which in turn necessitates the application of removable $t \mathrm{Bu}$ activating groups at the phenolic residues. ${ }^{19}$ Taken together, despite the tremendous developments in arylomycin synthesis, there is still no catalytic route towards these antibiotics that employs un-activated aromatic amino acids, in analogy to the natural pathway.

The biosynthetic machinery of the arylomycins was discovered in 2011 by Moore, Dorrestein and coworkers combining imaging mass spectrometry and genome mining. ${ }^{20}$ They identified a NRPS composed of the three proteins AryA, AryB and AryD and proposed it to assemble a linear lipopeptide precursor $\mathbf{3}$ (cf. Scheme 1A). Similar to glycopeptide biosynthesis, 3 was thought to undergo oxidative macrocyclization by the pathway-specific cytochrome P450 AryC while still bound to the terminal PCP of the NRPS, prior to thioesterase-mediated hydrolytic release of the product, e.g., arylomycin A2 (2). Interestingly, the NRPS does not contain an X domain, which is crucial for oxidative cross coupling in glycopeptide biosynthesis. Within this work, we thus set out to probe the potential of AryC as biocatalyst for the chemo-enzymatic assembly of $\mathbf{2}$, thereby also shedding light on its requirements for protein interaction partners to function.

Aiming for a convergent synthesis of the linear peptide precursor 3 while simultaneously avoiding peptide assembly and purification steps with the epimerization-prone HPG residue in place, the tripeptide portion (Scheme 1A, pink), ultimately composing the macrocycle of $\mathbf{2}$, was to be prepared by solid-phase peptide synthesis (SPPS). The lipopeptide side-chain (Scheme 1A, green) was synthesized by liquid phase peptide synthesis (LPPS). Preparation of the Grignard reagent from 1-bromo-3-methylbutane (4) using activated $\mathrm{Mg}$ turnings in THF with subsequent cuprate formation and addition to ethyl 7-bromoheptanoate (5) delivered ester 6 in $94 \%$ yield (Scheme 1B). ${ }^{21}$ Saponification of 6 with $\mathrm{NaOH}$ resulted in the required fatty acid building block 7 in $99 \%$ yield. Activation of 7 using $\mathrm{SOCl}_{2}$ and direct application of the resulting acid chloride for acylation of $\mathrm{O}-t \mathrm{Bu}-\mathrm{D}$-serine methyl ester, followed by mono $\mathrm{N}$-methylation of the amide gave ester $\mathbf{8}$ (combined $92 \%$ yield). Saponification of $\mathbf{8}$ in THF/LiOH delivered free acid $\mathbf{9}$ in 83\% yield. Compound 9 was coupled to dipeptide 10 (derived of $N$-Boc-D-alanine and glycine methyl ester in two steps, see SI) using EDCI and HOBt. Final saponification in THF/LiOH provided the lipopeptide side chain 11b (69\% yield over two steps). Overall, 11b was obtained in 7 linear steps and a yield of $49 \%$.

SPPS of the second building block commenced with commercially available 2-chloro-2-trityl resin preloaded $(0.4-1.0 \mathrm{mmol} / \mathrm{g})$ with $O$ - $t \mathrm{Bu}$-L-tyrosine (12). $\mathrm{N}$-Fmoc protection chemistry was employed for mild, non-racemizing conditions, ${ }^{22}$ with further reduction of the amounts of DBU $(0.5 \%)$ used during deprotection. Quantitative conversion of each individual SPPS step was controlled by cleavage of analytical amounts of product from the resin by treatment with 1\% TFA followed by HPLC analysis (for HPLC chromatograms, see Figure S1). Attachment of Fmoc-L-alanine was achieved using DIC and HOBt in DMF delivering 13 (Scheme 2). Fmoc removal (14) with DBU and attachment of Fmoc-L-HPG with COMU/NEt 3 in DMF furnished tripeptide 15. Removal of the Fmoc protection group (16) and installation of an $\mathrm{N}$-ortho-nitrobenzenesulfonyl (NBS) group (17) set the stage for selective mono $\mathrm{N}$-methylation to $\mathbf{1 8}$ following Kessler's protocol. ${ }^{23}$ Removal of NBS using mercaptoethanol liberated the amine 19, permitting installation of the lipopeptide chain $\mathbf{1 1 b}$ directly on resin to give $\mathbf{2 0 b}$

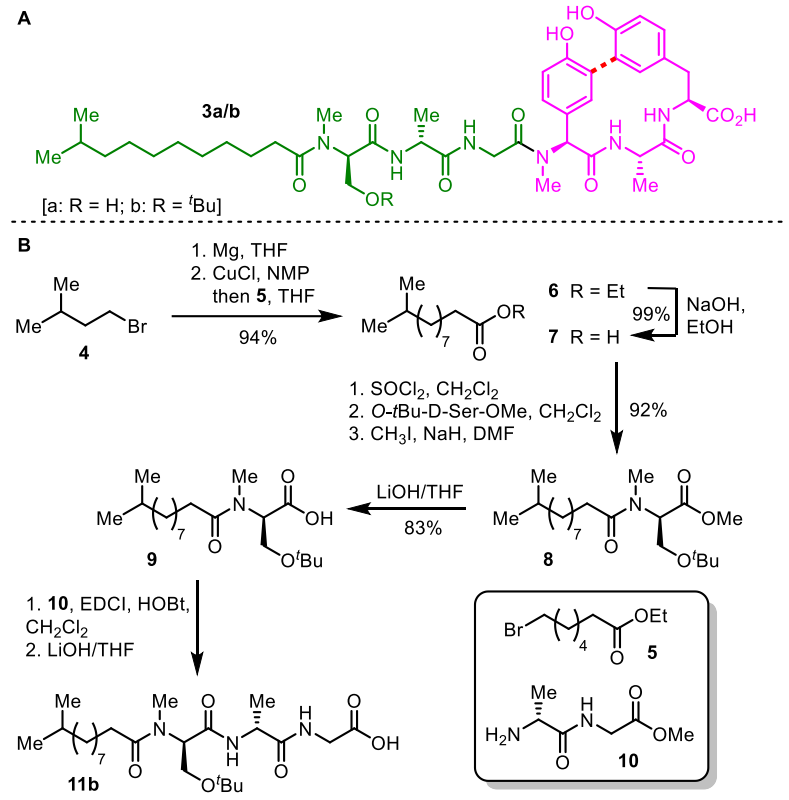

Scheme 1. A. Structure of the linear precursor peptide 3. Parts to be assembled by LPPS shown in green, by SPPS shown in pink. Dotted red line indicates the site of the strategic biocatalytic cross-coupling reaction using AryC. B. LPPS synthesis of the lipopeptide side chain $\mathbf{1 1 b}$.

Treatment of the resin with 9\% TFA (v/v) in $\mathrm{CH}_{2} \mathrm{Cl}_{2}$ released the product with concomitant $\mathrm{O} t \mathrm{Bu}$ deprotection at the Tyr residue to give the linear arylomycin A2 precursor $\mathbf{3 b}$. While simultaneous deprotection of the Ser-O $t \mathrm{Bu}$ group is possible upon longer treatment with TFA to directly deliver free $\mathbf{3 a}$, this also leads to significant epimerization. The fully unprotected arylomycin A2 precursor 3a was thus prepared by coupling of unprotected 11a (available by TFA-mediated deprotection of $\mathbf{1 1 b}$, see SI) to the still resinbound 19 to give 20a with subsequent release from the resin. Having precursors $\mathbf{3 a} / \mathbf{b}$ in hands, we next turned our attention to the production of recombinant AryC. The corresponding gene aryC was PCR-amplified from genomic DNA of the natural arylomycin producer strain Streptomyces roseosporus NRRL15998 (see SI for experimental details). Gene $a r y C$ was cloned into expression vector pHis8-TEV, permitting production of AryC with an $\mathrm{N}$-terminal octa-histidine tag for downstream affinity purification. Expression of AryC in E. coli BL21 and reconstitution of the purified recombinant enzyme using heminchloride ${ }^{24}$ with subsequent desalting and concentration applying centrifugal concentrators delivered AryC for enzymatic cross-coupling experiments.

Despite the expectation that AryC requires PCP-bound 3 for efficient substrate recognition - in analogy to all current examples of glycopeptide (bio)synthesis - we first probed its activity on free substrates, initially starting with $\mathbf{3 b}$. In these assays, the redox enzyme pair Fpr/PetF, ${ }^{7 b}$ which was also produced recombinantly in E. coli, was applied to shuttle electrons from the primary electron source NADPH to AryC. HPLC-MS analysis suggested very low turnover, further pointing at the potential PCP-dependency of AryC. Nevertheless, small amounts of putative product $\mathbf{2 b}$ were detectable by HPLC-MS. To our delight, simple exchange of the redox system to the mammalian adrenodoxin / adrenodoxin reductase system $\mathrm{Adx} / \mathrm{AdR}^{25}$ facilitated efficient biaryl coupling of the free acid precursor $\mathbf{3 b}$ to directly deliver arylomycin $\mathbf{2 b}$, without requirement for any further activation or tethering (Scheme 3 ). 


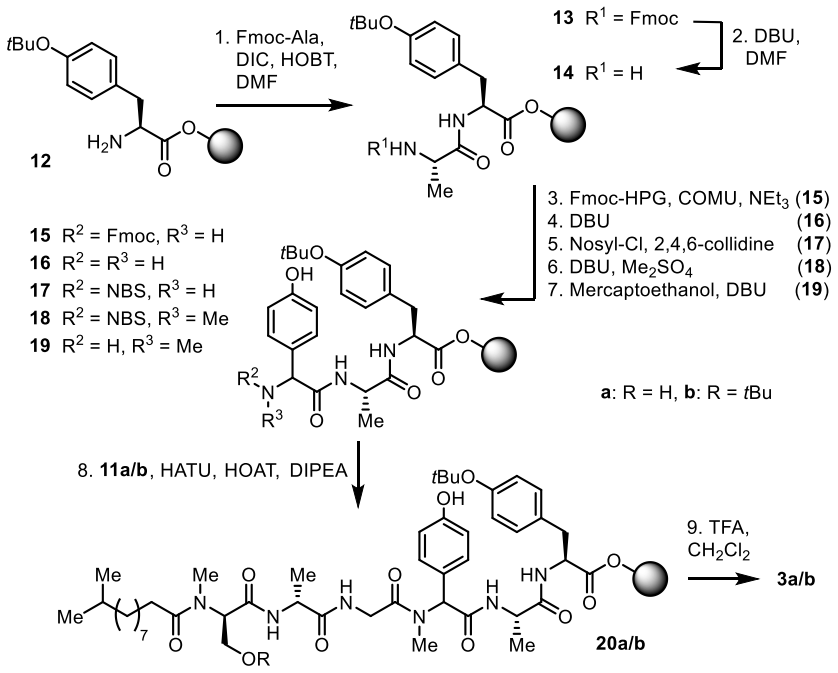

Scheme 2. Assembly of the linear arylomycin precursors $3 \mathbf{a} / \mathbf{b}$.

Identical activity was found for 3a to deliver arylomycin A2 (2a). Furthermore, additional application of recombinant glucose dehydrogenase GdhBM3 permitted in-situ recycling of $\mathrm{NADP}^{+}$from glucose. ${ }^{26}$ Overall, AryC is thus the first cytochrome $\mathrm{P} 450$ enzyme installing biaryl elements into NRPS-derived peptides to function without substrate-tethering and can thus efficiently be employed in the biocatalytic synthesis of arylomycin-type antibiotics.

Owing to this unprecedented activity of AryC on untethered, free substrates, we next aimed for its structural characterization by protein crystallography. Crystals of AryC fully reconstituted with hemin diffracted to $2.5 \AA$ and the structure was solved by molecular replacement taking advantage of the conserved core structure of $\mathrm{P} 450$ s, despite their generally low sequence conservation (for data processing and structure refinement statistics, see Supporting information; structure-based sequence alignment Figure S2). AryC shares the common, prism-like folding topology of P450s, characterized by a large $\alpha$-helical domain and a small $\beta$-sheet-rich domain, with the b-type heme cofactor sandwiched in between and deeply buried within the enzyme (Figure 2, Figure S3). The hemeiron is axially coordinated by the conserved, proximal Cys 355 residue, forming the iron-cysteinate bond, essential for the oxidation reaction carried out by this enzyme class. AryC shares about 35$40 \%$ sequence identity to other available P450s structures and can be superimposed with an r.m.s.d of 1.5-3 A (Figure S4).

The flexible loop that connects helices F and G (resi 170-190) and usually acts as a lid to the active site entrance of P450s is not resolved in the crystal structure of AryC. This loop is commonly disordered in most of the $\mathrm{P} 450$ structures reported to date and can be involved in interactions with peptidyl carrier proteins (PCP). ${ }^{27}$ Although the PCP carrier is not required for AryC-activity in vitro, as shown above, based on structural superposition of AryC with the two available $\mathrm{P} 450 \mathrm{~s}$ in complex with their respective substratebound PCPs allows to identify a potential binding site for the PCPcarrier at the $N$-terminus of helix G, the interface of the $N$-terminal loop region with the $C$-terminus of helix I and/or the $\beta 1$-sheet (Figure S5). Moreover, AryC contains the conserved PXXD-motif at the $N$-terminus of helix F.
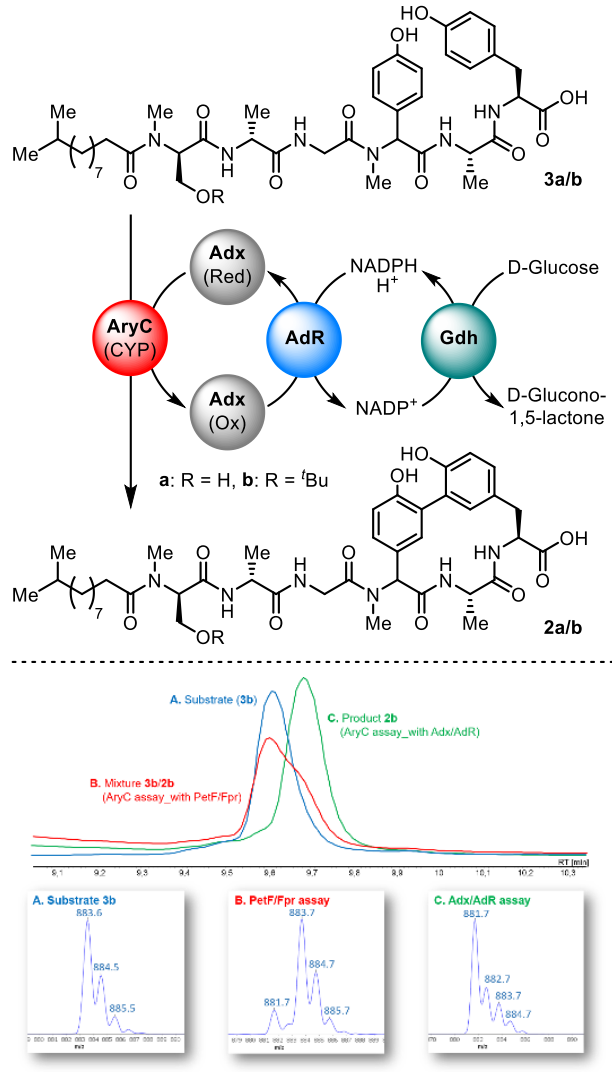

Scheme 3. Top: Biocatalytic cross-coupling of untethered, linear peptide precursors $\mathbf{3 a} / \mathbf{b}$ to arylomycin $\mathbf{2 a} / \mathbf{b}$ using AryC. Employed recombinant enzymes are depicted as colored spheres. Bottom: HPLC-MS analyses of the coupling reaction of $\mathbf{3 b}$ with different electron supply systems. Analyses of pure substrate (blue), reaction with Fpr/PetF (red) and Adx/AdR (green) are shown. $\mathrm{m} / z($ substrate $)=883.6 . \mathrm{m} / z$ (product $)=881.7$. For HRMS data and full HPLC-MS chromatograms, see SI.

A PRDD motif was shown to be responsible for the interaction with the $\mathrm{X}$-domain in the $\mathrm{P} 450 \mathrm{OxyB}$ catalyzing the first peptide cyclization step in vancomycin biosynthesis, ${ }^{8}$ and is present in AryC, despite the NRPS lacking such an X domain (Figure S5).

The funnel-shaped channel to the catalytic center is about $22 \AA$ long, and predominantly lined with aliphatic residues, resulting in a pronounced hydrophobic character (Figure 2, Figure S6). Substrate specificity and scope in P450s is determined by the accessibility of the catalytic heme-iron and fine-tuned by amino acid residues in helix I located above the proximal face of the heme ${ }^{8 b}$ (Figure 2A). Figure 2B shows a model for the possible binding mode of the HPG-Ala-Tyr-moiety of the substrate 3 to AryC, with the phenol rings of the HPG and Tyr-residues positioned over the heme face. The lipophilic tail of the substrate could thereby slot in the hydrophobic cavity (highlighted in green). This, in combination with the overall hydrophobic character of the entrance tunnel, most likely explains the ability of AryC to accept free lipopeptide substrates 3 , without additional PCP tethering. The interaction with the lipopeptide side-chain along with the available space of the hydrophobic funnel-shaped entrance channel to the AryC active site also explains the ability of this enzyme to even accept the unnatural, protected $\mathrm{O}-\mathrm{t} \mathrm{Bu}-\mathrm{Ser}$ synthetic analog $\mathbf{3 b}$. 


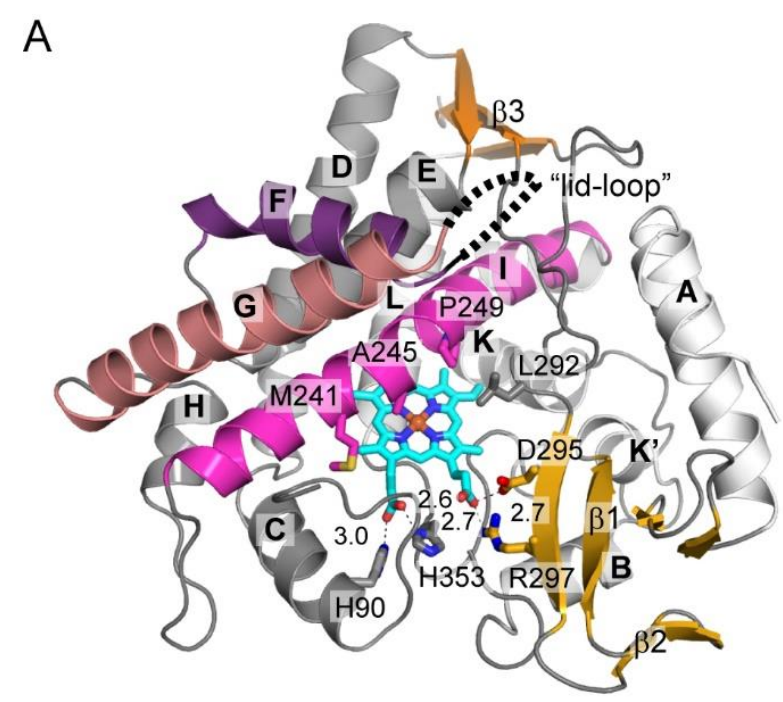

B
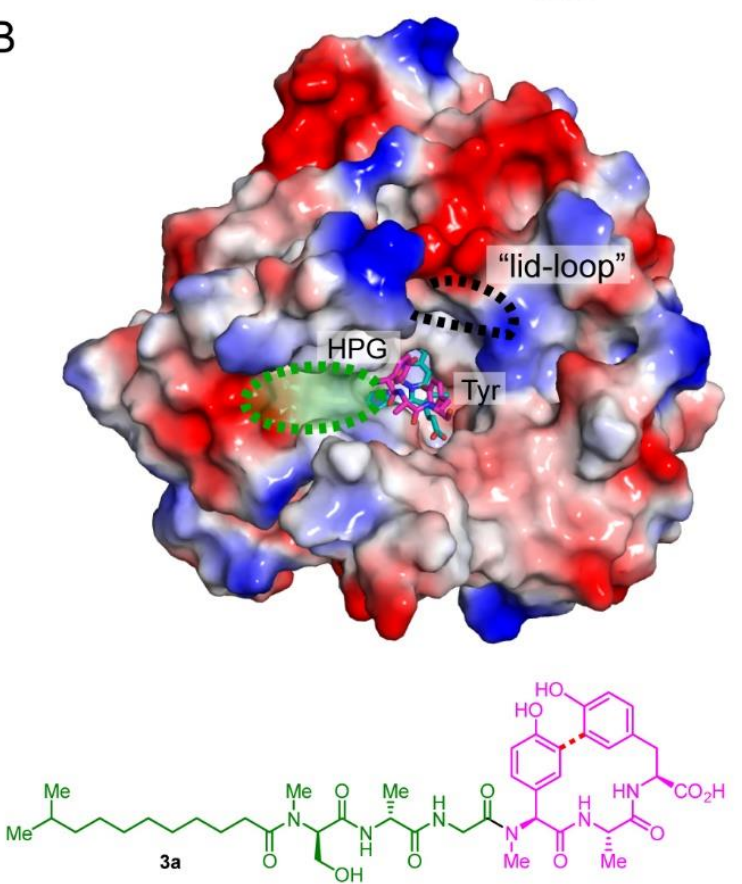

Figure 2. Structure of AryC. A. Overall folding topology of AryC displayed as ribbon. The heme and residues lining the active site are shown as stick model. Secondary structure elements are annotated as for P450s ( $\alpha$-helices A-L, $\beta$-sheets 1-3) and the flexible lid-loop is indicated by a dashed line. $\mathbf{B}$. Amino acid charges are mapped onto surface of AryC, highlighting the hydrophobic entrance channel to the active site. Proposed model for the binding-mode of the HPG-Ala-Tyr-moiety of the substrate (pink stick model) and likely positions of the lipophilic tail (green) are highlighted. The heme at the bottom of the active site is shown as cyan stick model.

Taken together, we have developed an efficient, convergent synthesis of arylomycin-type linear lipopeptide precursors $\mathbf{3}$ by combining liquid- and solid-phase peptide synthesis. Probing of the catalytic activity of AryC - the cytochrome P450 catalyzing biaryl formation in arylomycin biosynthesis - with $\mathbf{3}$ uncovered its unprecedented ability to accept these untethered, free precursors as substrates. Enzymatic cross-coupling by AryC thus for the first time establishes the biaryl structural element without the requirement of attaching precursor peptide substrates to PCP- or PCP-X interaction partners, thereby tremendously improving the practicability of such a chemo-enzymatic approach to BCPs. Moreover, the here provided structural and functional data could aid to modulate and adapt the substrate scope of AryC by site-directed mutagenesis of residues positioned around the heme-face and lining the substrate channel. This sets the stage for further future biocatalytic application of AryC in the synthesis of novel arylomycin analogs and provides a basis for exploring its application in the synthesis of aglycon structures of glycopeptide antibiotics.

\section{ASSOCIATED CONTENT}

\section{Supporting Information}

Experimental details, Figures S1-S32, analytical data.

The structure factors and model are deposited in the PDB (www.ebi.ac.uk/pdbe) under the accession code 7AYX.

\section{AUTHOR INFORMATION}

\section{Corresponding Author}

tobias.gulder@tu-dresden.de (T.A.M. Gulder)

sabine.schneider@cup.lmu.de (S. Schneider)

\author{
Author Contributions \\ $\|_{\text {These authors contributed equally. }}$
}

\section{ACKNOWLEDGMENT}

This project was generously funded by the German Research Foundation (DFG, GU 1233/1-1, SCHN 1273/6-1, Center for Integrated Protein Science Munich, CIPSM; EXC114). Shuangjie Shu, Hanna Hong and Tobias M. Milzarek thank the Chinese Scholarship Council (CSC), the Evonik Foundation, and the Stiftung der Deutschen Wirtschaft, respectively, for their PhD scholarships. We thank Prof. Rita Bernhardt (Saarland University, Saarbrücken) and Prof. Frank Schulz (Ruhr University Bochum) for kindly providing plasmid templates for amplification of adrladx (pBAR1607/pKKAdx) and $g d h$ (pACYCDuet-1::gdh-BM3), respectively.

\section{REFERENCES}

(1) (a) Bringmann, G.; Gulder, T.; Gulder, T. A. M.; Breuning, M. Atroposelective Total Synthesis of . Chem. Rev. 2011, 111, 563639. (b) Okano, A.; Isley, N. A.; Boger, D. L. Total Syntheses of Vancomycin-Related Glycopeptide Antibiotics and Key Analogues. Chem. Rev. 2017, 117, 11952-11993.

(2) Moore, M. J.; Qu, S.; Tan, C.;Cai, Y.; Mogi, Y.; Keith, D. J.; Boger, D. L. Next-Generation Total Synthesis of Vancomycin. J. Am. Chem. Soc. 2020, 142, 16039-16050.

(3) Al Toma, R. S.; Brieke, C.; Cryle, M. J.; Suessmuth, R. D. Structural aspects of phenylglycines, their biosynthesis and occurrence in peptide natural products. Nat. Prod. Rep. 2015, 32, 12071235.

(4) (a) Suessmuth, R. D.; Mainz, A. Nonribosomal Peptide Synthesis-Principles and Prospects. Angew. Chem. Int. Ed. Engl. 2017, 56, 3770-3821. (b) Hubbard, B. K.; Walsh, C. T. Vancomycin assembly: nature's way. Angew. Chem. Int. Ed. Engl. 2003, 42, 730-765. (c) Suessmuth, R. D.; Wohlleben, W. The biosynthesis of glycopeptide antibiotics--a model for complex, non-ribosomally synthesized, peptidic secondary metabolites. Appl. Microbiol. Biotechnol. 2004, 63, 344-350. 
(5) McCormick, M. H.; Stark, W. M.; Pittenger, G. E.; Pittenger, R. C.; McGuire, J. M. Vancomycin, a new antibiotic. I. Chemical and biologic properties. Antibiot. Annu. 1955, 56, 606-611.

(6) (a) Bischoff, D.; Pelzer, S.; Holtzel, A.; Nicholson, G. J.; Stockert, S.; Wohlleben, W.; Jung, G.; Suessmuth, R. D. The Biosynthesis of Vancomycin-Type Glycopeptide Antibiotics-New Insights into the Cyclization Steps. Angew. Chem. Int. Ed. 2001, 40, 16931696. (b) Bischoff, D.; Pelzer, S.; Bister, B.; Nicholson, G. J.; Stockert, S.; Schirle, M.; Wohlleben, W.; Jung, G.; Suessmuth, R. D. The Biosynthesis of Vancomycin-Type Glycopeptide Antibiotics-The Order of the Cyclization Steps. Angew. Chem. Int. Ed. 2001, 40, 4688-4691. (c) Pelzer, S.; Suessmuth, R. D.; Heckmann, D.; Recktenwald, J.; Huber, P.; Jung, G.; Wohlleben, W. Identification and Analysis of the Balhimycin Biosynthetic Gene Cluster and Its Use for Manipulating Glycopeptide Biosynthesis in Amycolatopsis mediterranei DSM5908. Antimicrob. Agents Chemother. 1999, 43, 1565-1573. (d) Stegmann, E.; Pelzer, S.; Bischoff, D.; Puk, O.; Stockert, S.; Butz, D.; Zerbe, K.; Robinson, J.; Suessmuth, R. D.; Wohlleben, W. Genetic analysis of the balhimycin (vancomycin-type) oxygenase genes. J. Biotechnol. 2006, 124, 640-653. (e) Recktenwald, J.; Shawky, R.; Puk, O.; Pfennig, F.; Keller, U.; Wohlleben, W.; Pelzer, S. Nonribosomal biosynthesis of vancomycin-type antibiotics: a heptapeptide backbone and eight peptide synthetase modules. Microbiology. 2002, 148, 1105-1118. (f) Bischoff, D.; Bister, B.; Bertazzo, M.; Pfeifer, V.; Stegmann, E.; Nicholson, G. J.; Keller, S.; Pelzer, S.; Wohlleben, W.; Suessmuth, R. D. The biosynthesis of vancomycin-type glycopeptide antibiotics-a model for oxidative side-chain cross-linking by oxygenases coupled to the action of peptide synthetases. ChemBioChem. 2005, 6, 267-272.

(7) (a) Zerbe, K.; Woithe, K.; Li, D. B.; Vitali, F.; Bigler, L.; Robinson, J. A. An oxidative phenol coupling reaction catalyzed by oxyB, a cytochrome $\mathrm{P} 450$ from the vancomycin-producing microorganism. Angew. Chem. Int. Ed. 2004, 43, 6709-6713. (b) Woithe, K.; Geib, N.; Zerbe, K.; Li, D. B.; Heck, M.; Fournier-Rousset, S.; Meyer, O.; Vitali, F.; Matoba, N.; Abou-Hadeed, K.; Robinson, J. A. Oxidative phenol coupling reactions catalyzed by OxyB: a cytochrome $\mathrm{P} 450$ from the vancomycin producing organism. implications for vancomycin biosynthesis. J. Am. Chem. Soc. 2007, 129, 6887-6895. (c) Woithe, K.; Geib, N.; Meyer, O.; Wortz, T.; Zerbe, K.; Robinson, J. A. Exploring the substrate specificity of OxyB, a phenol coupling $\mathrm{P} 450$ enzyme involved in vancomycin biosynthesis. Org. Biomol. Chem. 2008, 6, 2861-2867. (d) Geib, N.; Woithe, K.; Zerbe, K.; Li, D. B.; Robinson, J. A. New insights into the first oxidative phenol coupling reaction during vancomycin biosynthesis. Bioorg. Med. Chem. Lett. 2008, 18, 3081-3084. (e) Li, D. B.; Woithe, K.; Geib, N.; Abou-Hadeed, K.; Zerbe, K.; Robinson, J. A. Chapter 19. In vitro studies of phenol coupling enzymes involved in vancomycin biosynthesis. Methods Enzymol. 2009, 458, 487509. (f) Li, D. B.; Robinson, J. A. An improved solid-phase methodology for the synthesis of putative hexa- and heptapeptide intermediates in vancomycin biosynthesis. Org. Biomol. Chem 2005, 3, 1233-1239. Die markierten sind aus meiner Diss

(8) (a) Haslinger, K.; Peschke, M.; Brieke, C.; Maximowitsch, E.; Cryle, M. J. X-domain of peptide synthetases recruits oxygenases crucial for glycopeptide biosynthesis. Nature. 2015, 521, 105-109. (b) Peschke, M.; Gonsior, M.; Suessmuth, R. D.; Cryle, M. J. Understanding the crucial interactions between Cytochrome P450s and non-ribosomal peptide synthetases during glycopeptide antibiotic biosynthesis. Curr. Opin. Struct. Biol. 2016, 41, 46-53.

(9) (a) Forneris, C. C.; Seyedsayamdost, M. R. In Vitro Reconstitution of OxyC Activity Enables Total Chemoenzymatic Syntheses of Vancomycin Aglycone Variants. Angew. Chem. Int. Ed. 2018 , 37, 8048-8052. (b) Tailhades, J.; Zhao, Y.; Schoppet, M.; Greule,
A.; Goode, R. J. A.; Schittenhelm, R. B.; De Voss, J. J.; Cryle, M. J. Enzymatic Cascade To Evaluate the Tricyclization of Glycopeptide Antibiotic Precursor Peptides as a Prequel to Biosynthetic Redesign. Org. Lett. 2019, 21, 8635. (c) Zhao, Y.; Goode, R. J. A.; Schittenhelm, R. B.; Tailhades, J.; Cryle, M. J. Exploring the Tetracyclization of Teicoplanin Precursor Peptides through Chemoenzymatic Synthesis. J. Org. Chem. 2020, 85, 1537-1547. (d) Tailhades, J.; Zhao, Y.; Ho, Y. T. C.; Greule, A.; Ahmed, I.; Schoppet, M.; Kulkarni, K.; Goode, R. J. A.; Schittenhelm, R. B.; De Voss, J. J.; Cryle, M. J. A Chemoenzymatic Approach to the Synthesis of Glycopeptide Antibiotic Analogues. Angew. Chem. Int. Ed. 2020, 59, 10899-10903.

(10)Zhao, Y.; Ho, Y. T. C.; Tailhades, J.; Cryle M. J. Understanding the Glycopeptide Antibiotic Crosslinking Cascade: In Vitro Approaches Reveal the Details of a Complex Biosynthesis Pathway. ChemBioChem. 2020, 21, 1-10 (Early View).

(11) Schimana, J; Gebhardt, K.;Höltzel, A.; Schmid, D. G.; Suessmuth, R.; Müller, J.; Pukall, R.; Fiedler, H.-P. Arylomycins A and B, New Biaryl-bridged Lipopeptide Antibiotics Produced by Streptomyces sp. Tü 6075. J. Antibiot. 2002, 55, 565-570.

(12) (a) Kulanthaivel, P.; Kreuzman, A. J.; Strege, M. A.; Belvo, M. D.; Smitka, T. A.; Clemens, M.; Swartling, J. R.; Minton, K. L.; Zheng, F.; Angleton, E. L.; Mullen, D.; Jungheim, L. N.; Klimkowski, V. J.; Nicas, T. I.; Thompson, R. C.; Peng, S.-B. Novel lipoglycopeptides as inhibitors of bacterial signal peptidase I. J. Biol. Chem. 2004, 279, 36250-36258. (b) Paetzel, M.; Goodall, J. J.; Kania, M.; Dalbey, R. E.; Page, M. G. P. Crystallographic and biophysical analysis of a bacterial signal peptidase in complex with a lipopeptide-based inhibitor. J. Biol. Chem. 2004, 279, 3078130790. (c) Luo, C.; Roussel, P.; Dreier, J.; Page, M. G. P.; Paetzel, M. Crystallographic Analysis of Bacterial Signal Peptidase in Ternary Complex with Arylomycin A2 and a $\beta$-Sultam Inhibitor. Biochemistry 2009, 48, 38, 8976-8984. (d) Smith, P. A.; Romesberg, F.E. Mechanism of action of the arylomycin antibiotics and effects of signal peptidase I inhibition. Antimicrob. Agents Chemother. 2012, 56, 5054-5060. (e) Walsh, S.; Peters, D. S.; Smith, P. A.; Craney, A.; Dix, M. M.; Cravatt, B. F.; Romesberg, F. E. Inhibition of Protein Secretion in Escherichia coli and Sub-MIC Effects of Arylomycin Antibiotics. Antimicrob. Agents Chemother. 2019, 63, 1-13oder e01253-18.

(13) (a) Roberts, T. C.; Smith, P. A.; Cirz, R. T.; Romesberg, F. E. Structural and initial biological analysis of synthetic arylomycin A2. J. Am. Chem. Soc. 2007, 129, 15830-15838. (b) Dufour, J.; Neuville, L.; Zhu, J. Total Synthesis of Arylomycin A2, a Signal Peptidase I (SPase I) Inhibitor. Synlett 2008, 2355-2359. (c) Dufour, J.; Neuville, L.; Zhu, J. Intramolecular Suzuki-Miyaura reaction for the total synthesis of signal peptidase inhibitors, arylomycins $\mathrm{A}(2)$ and $\mathrm{B}(2)$. Chemistry 2010, 16, 10523-10534. (d) Liu, J.; Luo, C.; Smith, P. A.; Chin, J. K.; Page, M. G. P.; Paetzel, M.; Romesberg, F. E. Synthesis and characterization of the arylomycin lipoglycopeptide antibiotics and the crystallographic analysis of their complex with signal peptidase. J. Am. Chem. Soc. 2011, 133, 17869-17877.

(14) (a) Roberts, T. C.; Schallenberger, M. A.; Liu, J.; Smith, P. A.; Romesberg, F. E. Initial efforts toward the optimization of arylomycins for antibiotic activity. J. Med. Chem. 2011, 54, 4954-4963. (b) Liu, J.; Smith, P. A.; Steed, D. B.; Romesberg, F. Efforts toward broadening the spectrum of arylomycin antibiotic activity. Bioorg. Med. Chem. Lett. 2013, 23, 5654-5659.

(15) Smith, P. A.; Koehler, M. F. T.; Girgis, H. S.; Yan, D.; Chen, Y.; Chen, Y.; Crawford, J. J.; Durk, M. R.; Higuchi, R. I.; Kang, J.; Murray, J.; Paraselli, P.; Park, S.; Phung, W.; Quinn, J. G.; Roberts, T. C.; Rougé, L.; Schwarz, J. B.; Skippington, E.; Wai, J.; Xu , M.; 
Yu, Z.; Zhang, H.; Tan, M.-W.; Heise, C. E. Optimized arylomycins are a new class of Gram-negative antibiotics. Nature 2018, $561,189-194$.

(16) Lim, N.-K.; Linghu, X.; Wong, N.; Zhang, H.; Sowell, C. G.; Gosselin, F. Macrolactamization Approaches to Arylomycin Antibiotics Core. Org. Lett. 2019, 21, 147-151.

(17) Wong, N.; Petronijević, F.; Hong, A. Y.; Linghu, X.; Kelly, S. M.; Hou, H.; Cravillion, T.; Lim, N.-K.; Robinson, S. J.; Han, C.; Molinaro, C.; Sowell, C. G.; Gosselin, F. Stereocontrolled Synthesis of Arylomycin-Based Gram-Negative Antibiotic GDC-5338. Org. Lett. 2019, 21, 9099-9103.

(18) Peters, D. S.; Romesberg, F. E.; Baran, P. S. Scalable Access to Arylomycins via C-H Functionalization Logic. J. Am. Chem. Soc. 2018, 140, 2072.

(19) Ben-Lulu, M.; Gaster, E.; Libman, A.; Pappo, D. Synthesis of Biaryl-Bridged Cyclic Peptides via Catalytic Oxidative Cross-Coupling Reactions. Angew. Chem. Int. Ed. Engl. 2020, 59, 4835-4839.

(20) Liu, W. T.; Kersten, R. D.; Yang, Y.-L.; Moore, B. S.; Dorrestein, P. C. Imaging mass spectrometry and genome mining via short sequence tagging identified the anti-infective agent arylomycin in Streptomyces roseosporus. J. Am. Chem. Soc. 2011, 133, 18010-18013.

(21) Cahiez, G.; Chaboche C.; Jézéquel M. Cu-Catalyzed Alkylation of Grignard Reagents: A New Efficient Procedure. Tetrahedron 2000, 56, 2733-2737.

(22)Brieke C.; Cryle, M. J. A Facile Fmoc Solid Phase Synthesis Strategy To Access Epimerization-Prone Biosynthetic Intermediates of Glycopeptide Antibiotics. Org. Lett. 2014, 16, 2454-2457. (23) Biron, E.; Chatterjee, J.; Kessler, H. Optimized selective Nmethylation of peptides on solid support. J. Peptide Sci. 2006, 12, 213-219.

(24) Schneider, S.; Sharp, K. H.; Barker, P. D.; Paoli, M. An induced fit conformational change underlies the binding mechanism of the heme transport proteobacteria-protein HemS. J. Biol. Chem. 2006, 281, 32606-32610.

(25) Ewen, K. M.; Ringle, M.; Bernhardt, R. Adrenodoxin--a versatile ferredoxin. IUBMB Life. 2012, 64, 506-512.

(26) Weckbecker, A.; Hummel, W. Glucose dehydrogenase for the regeneration of NADPH and NADH in Microbial Enzymes and Biotransformations (Ed.: Barredo, J. L.), Humana Press, Totowa, NJ, 2005, 225-238.

(27) (a) Cryle, M. J.; Schlichting I. Structural insights from a P450 Carrier Proteincomplex reveal how specificity is achieved in the P450BioIACP complex. PNAS 2008, 105, 15696-15701. (b) Haslinger, K.; Brieke, C.; Uhlmann, S.; Sieverling, L.; Süssmuth, R. D.; Cryle, M. J. The structure of a transient complex of a nonribosomal peptide synthetase and a cytochrome $\mathrm{P} 450$ monooxygenase. Angew. Chem. Int. Ed. Engl. 2014, 53, 8518-8522. 